\title{
Research of Mechanical-Arm Motion Control Algorithm based on Neutral Network
}

\author{
Wenjun $\mathrm{Xu}^{1}{ }^{1}$ Lan $\mathrm{Chen}^{2}$, Yuzhen $\mathrm{Lin}^{1}{ }^{1}$,Wenjun Liu ${ }^{1}$ and Fan Yang ${ }^{1}$ \\ ${ }^{1}$ (Quzhou College Of Technology, Quzhou 324000,China) \\ zjqzxwj@sina.com \\ ${ }^{2}$ (Zhejiang Wanliyang Transmission Co.,Ltd.Jinhua 321000,China) \\ zjqzxwj@sina.com
}

\begin{abstract}
Currently, robots have been widely used in all walks of life, and mechanical arm of robots have always been the focus of research in the aspect of control. This paper first analyzes motions of mechanical arm, and then appliedneutrd network in the analysis of mechanical arms' motions, effectively solving the issue that mechanical arms have multiple variables with strong coupling. Finally, it conducts the optimal control over mechanical arms' motion through genetic algorithm. Simulation experiment shows that this method is effective and can improve effects of mechanieal arms' motions.
\end{abstract}

Keywords: neutral network, mechanical arm, gene(ic) algorithm

\section{Introduction}

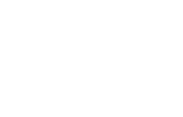

Currently, the robotic technology is widely used in various areas of national life, from the high-end special robots to civilian home robots, which has raised higher requirements for various dynamic performance of the robotic controlling system. As the classic representative of industrial robots, mechanical arms are also equipped with the kinematic and dynamic characteristics of general robots, that is, multivariate, time-varying, highly coupling and nonlinear,etc (11]

Domestic and forefgny scholars have studied the literature [2] somatosensory manipulator control agorithm based on comfort. Tracking and remote grasping objects experiment by the operation, the measured starting a comfort control algorithm relative error of the robot in less than $5 \%$, the tip position of absolute error less than $2 \mathrm{~mm}$, comfort increase $51.8 \%$ than the traditional control algorithms, to meet the somatosensory control accuracy Based on the large extent to ensure the user's comfort and improve the somatosensory control efficiency. Literature [3] proposed a new type of flexible manipylator based on fuzzy control trajectory tracking and vibration control strategy. First derivation for PD flexible arm angle control Parallel fuzzy controller, which was subsequently improved, a new non- Parallel fuzzy controller, combined with input shaping policy, derived hybrid fuzzy controller is a new type of flexible manipulator. The experimental results show that the new fuzzy controller better able to complete the proposed flexible manipulator trajectory tracking task and significantly reduce the vibration of flexible manipulator. Literature [4] proposed an adaptive fuzzy control and non-singular terminal sliding mode control of a combination of tracking control method. This method is based on the non-holonomic mobile manipulators dynamics model based on the use of nonlinear fuzzy Gaussian function neural network approximation performance, optimization compensate conventional method in a mobile manipulator system, the system is difficult to solve the unknown parameter uncertainty, and through the application of non-singular terminal sliding mode control to eliminate unknown outside interference and fuzzy control approximation errors on the system and improve 
the robustness and control performance of the system. Literature [5] using the second Lagrange equations establish space manipulator system dynamics model based on a collision course with the acquisition of satellite space manipulator momentum conservation principle, the use of momentum impulse method to calculate the impact of the collision of space manipulator motion Effects state, which will cause instability Effects motion of space manipulator system and the flexible rod elastic vibration. Literature [6] proposed on the basis of floating space manipulator dynamics equations established on the calculation of the shock effect of the contact arm to capture the target by the collision process. Simultaneous space manipulator dynamics equations and objectives established after capture kinetic equation with the target mix of space manipulator system through numerical simulation reveals the influence of collision impact effect on the mix of motion of the system status and to verify the above-mentioned control effectiveness of the method. Literature [7] proposed PID parameters using genetic algorithm tuning space manipulator control algorithm can effectivelyimprove the accuracy of the joint angle control. The simulation results show that the algorithm uses a genetic algorithm tuning PID parameters for space manipulator joint angle has better control performance, and overcome the traditional shortcomings of PID tuning parameters difficult.

In view of the disadvantages of multi variable and strong doupling in the space manipulator, this paper firstly analyzes the motion of the space manipulator, and then uses the neural network to analyze the manipulator motion. The simulation experiment shows that the method is effective and can improve the movement effect of the robot arm.

\section{Analysis of MechanicakAms' Motions}

The robot arm is an open chain multi-link mechanism, which is fixed by a fixed base, and is installed at the free end to achieve the purpose of the work. Between two connecting rod is connected by a rotating foint or a movable joint, and the joint is driven by the movement of the connecting rod.

\subsection{Spatial pescription and Coordinate Transformation}

The position and orientafion of the space point includes the position and direction. The position and direction of any point in space can be expressed by a pose description. The position $P^{A}$ of anypoint $P$ in space at a right angle coordinate system $\{A\}$, the formula is as follows:

Among them, $p_{x}, p_{y}, p_{z}$ is the component $P$ vector coordinate in the

$$
P^{A}=\left[p_{x}, p_{y}, p_{z}\right]^{T}
$$

A) rectangular coordinate system, in which $P^{A}$ is the position vector. The coordinates are rotated to obtain the following formula (2)

$$
{ }_{B}^{A} R=\left[{ }^{A} x_{B},{ }^{A} y_{B},{ }^{A} z_{B}\right]=\left[\begin{array}{lll}
r_{11} & r_{12} & r_{13} \\
r_{21} & r_{22} & r_{23} \\
r_{31} & r_{32} & r_{33}
\end{array}\right]
$$

Called the rotation matrix. $\mathrm{X}, \mathrm{Y}$ and $\mathrm{Z}$ axes are rotated to rotate $\theta$, and the $\mathrm{S}$ is expressed by sin; the $\mathrm{C}$ is expressed by $\cos$, and the rotation matrix is the formula (3). 


$$
\begin{aligned}
& R(x, \theta)=\left[\begin{array}{ccc}
1 & 0 & 0 \\
0 & \cos \theta & -\sin \theta \\
0 & \sin \theta & \cos \theta
\end{array}\right] \\
& R(y, \theta)=\left[\begin{array}{llc}
\cos \theta & 0 & \sin \theta \\
0 & 1 & 0 \\
-\sin \theta & 0 & \cos \theta
\end{array}\right] \\
& R(y, \theta)=\left[\begin{array}{lll}
\cos \theta & -\sin \theta & 0 \\
\sin \theta & \cos \theta & 0 \\
0 & 0 & 1
\end{array}\right]
\end{aligned}
$$

By using the coordinate transformation, the spatial point $\mathrm{P}$ can be realized in different coordinate system. Coordinate transformation is usually included in the translation and rotation transformation. When the two coordinate system $\{B\}$ and $\{A\}$ the orientation of the same but the origin of different, then relative to the coordinate system $\{A\}$, the coordinates $\{B\}$ of any point $\mathrm{P}$ position vector can be deseribed as formula (4), if the origin of the two coordinates of the same but different, for any point $P$ in the two coordinate system $\{A\}$ and $\{B\}$ the descriptign ${ }^{A} P$ and ${ }^{B} P$ transformation formula (5)

$$
\int \ell_{A}^{A} P={ }_{B}^{A} R{ }^{A} P+P_{Y} P_{B_{O}}
$$

\subsection{Equation of Mechanical Arms' Motions}

The study of the kineniatics of the manipulator is closely related to the establishment of the coordinate system. The specific requirements are, for each link of the robot arm, respectively, the establishment of a connecting tod coordinate system, the establishment of a certain position between the various coordinates, these relations can be used to describe the spatial position and posture of the egnnecting rod, thereby obtaining the manipulator position and posture of the manipulatorend, as shown in Figure 1.

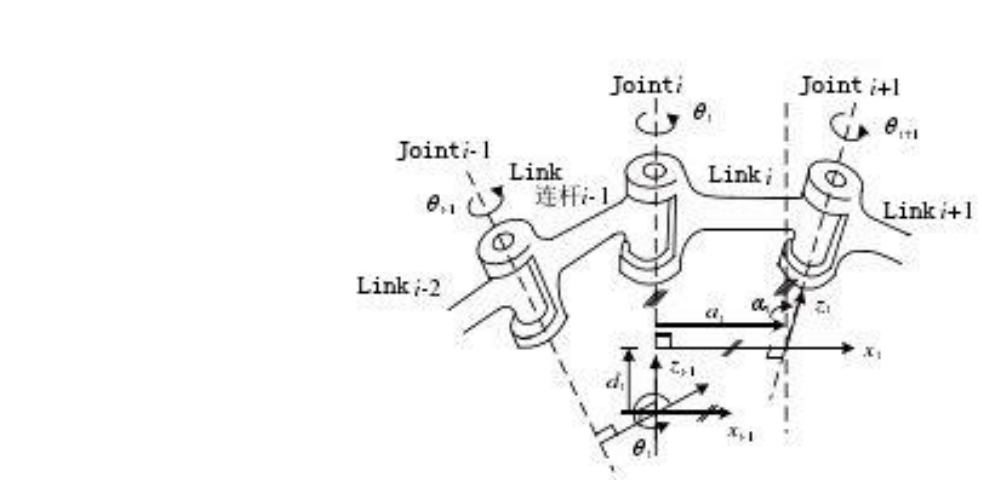


Figure 1. Relation of Mechanical Arms' Location

\begin{tabular}{|c|c|}
\hline Name & Significance \\
\hline$a_{i}$ & Length of the i connecting rod \\
\hline$\alpha_{i}$ & Twist angle of the i connecting rod \\
\hline$d_{i}$ & $\begin{array}{c}\text { Distance between the i connecting rod and the } \\
\text { i }+1 \text { connecting rod }\end{array}$ \\
\hline$\theta_{i}$ & $\begin{array}{c}\text { Twist Angle between the } \mathrm{i} \text { connecting rod and } \\
\text { the } \mathrm{i}+1 \text { connecting rod }\end{array}$ \\
\hline
\end{tabular}

Therefore, relation of motions can be expressed as:

$$
{ }^{i} T_{i-1}=R o\left(t, Q_{i}\right) \operatorname{Tr} a(n 0 s, \varphi, d) \operatorname{Tr} a\left(n s, \theta, 0 \not \chi_{i} K\right.
$$

According to the actual needs, the formula (6) is transformed into two bar linkage, such as formula (7).

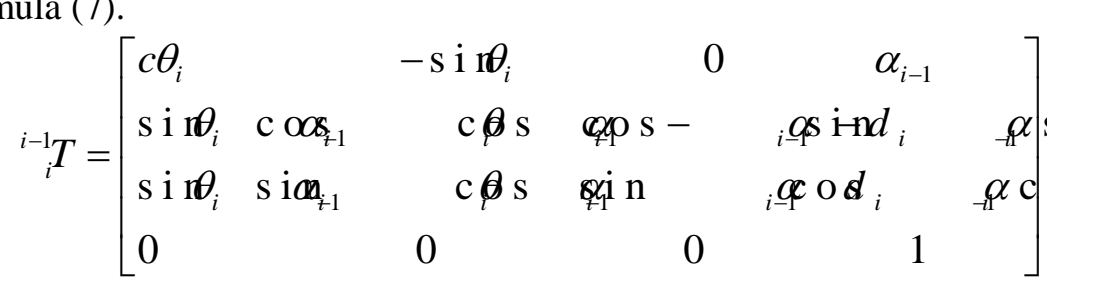

\section{Neutral Network}

\subsection{Definition of Neutral Network}

BP neural network is a kind of one-way transmission network, which is usually composed of input layer, hideden layer and oytput layer. It will signal the forward pass and the back propagation. The input and butput of each layer is calculated by the formula (8) in the forward pass. When the output layer is less than the desired output, the output is transmitted to the back propagation, and the weights and thresholds are adjusted according to the error between the expectation and the actual output. The formula for the adjustment of weight values(9).

$$
\left\{\begin{array}{l}
W_{i}=\sum_{j} w_{i j} x_{j}+\theta_{i} \\
y_{i}=f\left(W_{i}\right)
\end{array}\right.
$$

In formula $(8), W_{i}$ is the activated value of node at level $i, \theta_{i}$ is the threshold value, $x_{j}$ is the mput signal, $w_{i j}$ is the connecting coefficient between node $i$ and node $j$, and $y_{i}$ is the export value of node $i$.

$$
w_{i j}(t+1)=w_{i j}(t)+\frac{\partial E}{\partial w_{i j}}
$$

In formula (9), $\frac{\partial E}{\partial w_{i j}}$ refers to the difference between expected output and actual output of neutral network.

\subsection{Neutral Network Training}

Suppose the input mode is $x_{l}^{i}, i \in\{1,2, \cdots k\} . k$ is the number of input data, $n$ is the input of the input pattern, and $w_{l}^{m}$ is the dimension of the input pattern, $w_{l}^{m}$ is the right of the $l$ input layer to the hidden layer of the $m$ neutron. 


$$
\partial^{m}=f\left(\sum_{l=1}^{n}\left(x_{l}^{i} w_{l}^{m}\right)-T^{m}\right)
$$

In the formula, $f(x)$ is the activated function of neutron at the hidden level. In this paper, $f(x)$ is the hyperbolic tangent function, $f(x)=1-\frac{2 e^{-x}}{e^{x}+e^{-x}}, T^{m}$ refers to the layout of the $\mathrm{m}$ neutron at the hidden level, reflecting the larger activator value on the minor range [-1,1]. Once neutrons at the hidden level are calculated, the output value of

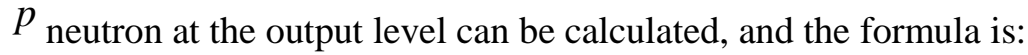

$$
S_{p}^{i}=\sigma\left(\sum_{m=1}^{h} \partial v_{p}^{m}\right)
$$

In the formula, $\sigma(x)$ refers to the activated function of neutrons at the output level, and $\sigma(x)=\frac{1}{1+e^{-x}}$. It reflects the bigger activated value or the minor range $[0,1] . v_{p}^{m}$ refers to the connection right of $m$ neutron at the hidaen level to the neutron at the output level.

\section{Research Based on Improved BP Neutral Network Algorithm in Mechanical Arms}

\subsection{Proposition of Problems}

Because the search of the genetic algorithm does not depend on gradient information, it is not required to solve the problem. It is ony needed to solve the fitness function under the constraint conditions. The optimization problem of genetic neural network is described as follows?

$$
E_{k=1} \sum_{t=1}^{\hat{N}_{t}} \sum_{t}^{n}\left[y_{k}(t)-\hat{y}_{k}(t)\right]
$$

Herein, $E_{1}$ relers to the total errors of the network, $\hat{y}_{k}(t)$ refers to the theoretical output of network, and $\hat{y}_{k}(t)$ refers to the actual output of network. Herein, $\hat{y}_{k}(t)$ is as follows:

$$
E_{2}=\frac{\sum_{k=1}^{N_{1}} \sum_{t=1}^{n}\left[y_{k}(t)=\sum_{i=1}^{m} w_{i j} \llbracket \hat{y}_{i}(t)\right]}{N-N_{1}} \text { as test sample average mean square error. Express }
$$

network output data to estimate reliability. The value of $E_{2}$ is small, the output represents a large number of network reliability, on the contrary, the network output is not much reliable.

\subsection{Design of Algorithm}

Algorithm by using genetic algorithms in the basic solution space initially identified by the BP network (network connection weights and neuron threshold value range), through genetic selection, crossover and mutation operation on the sample individual merit continued evolution until evolving to the $k$ (evolution generation by the initial total given) generation, selecting individuals to adapt to the individual to determine the degree 
of the largest network of structures and networks of initial weights and thresholds.

(1) Determine the Range of Basic Solutions

Three BP network is used to determine the basic solution space. Firstly, network's training times and training error $\varepsilon_{1}$ are set, input the training sample for training, and then input the test samples to get error $\varepsilon_{2}$. When both error $\varepsilon_{1}$ and $\varepsilon_{2}$ are relatively satisfactory, set the maximum value and minimum value in the connecting value as $u_{\max }$ and $u_{\min }$ respectively with the range $\left[u_{\min }, u_{\max }\right]$ as the basic solution space of connecting right.

(2) Calculation of Adaptation Function

In genetic algorithm, the only requirement is that the input can be calculated to be more non negative results. Therefore, the error function of the network is used as fitness function.

To calculate the fitness of each individual in the group, the number of hidden nodes is obtained by the switching coefficient. The network is connected with the network by the weight of the network, and the fitness of each individud is calculated agcording to (14).

(3) Crossover and Mutation Factor

Cross the selected individuals with $P_{c}$ as the probability, and suppose the individual $i$ and individual $i+1$ are crossed while the cross operator is as follows:

$$
\left\{\begin{array}{l}
x_{i}^{t+1}=c_{1}-\left(1-c_{i}\right) x_{i+1}^{t^{t}} \\
x_{i+1}^{t+1}-\left(1-c_{i}\right) \square x_{i}^{0}+x_{i+1}
\end{array}\right.
$$

Herein, $x_{i}^{t}$ and $x_{i+1}^{t}$ are a pair of individuals before cross, $x_{i}^{t+1}$ and $x_{i+1}^{t+1}$ are a pair of individuals after cross, while $c_{i}$ is a random number between 0 and 1. Mutate individuals with $p_{m}$ as the probability, and surpose the mutation is conducted to individual $i$. Mutation operator is shown as follofws:

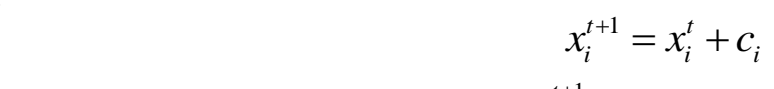

$x_{i}^{t}$ refers toindividuals before mutation, $x_{i}^{t+1}$ refers to individuals after mutation, and $c_{i}$ is a random number distributed evenly to guarantee mutated individuals are still within the range of search

\subsection{Algorithím Steps}

Step PThe mechanical arm movement of each joint and connecting rod sequence $(x, y)$, sequence $\left\{T_{1}, T_{2}, \cdots T_{n}\right\}$ was generated according to formula (7)

Step 2: Using neural network parallel search mechanism of genetic algorithm, the chromosome coding for all $a_{i}(k)(i=1,2, \cdots, N) \quad N$ dimensional vectors $a(k)=\left[a_{1}(k), a_{2}(k), \cdots a_{n}(k)\right]^{T}$

Step 3: produce initial solutions formed by $N$ possible solutions $a_{i}(k)$, initialize the $N$ components of $a_{i}(k)$ as zero mean value, the variance of 1 standard normal distribution random number

Step 4: calculate the adaptation according to formula (14)

Step 5: Through the selection, crossover and mutation operators to generate new species, the selection of a certain number of individuals $a_{1}(k), a_{2}(k), \cdots a_{i}(k)$ and based on the combination of more than rules that make up a new generation of individuals $a_{1}(k+1), a_{2}(k+1), \cdots a_{i}(k+1)$. 
Step 6: Repeat step 4-5 to get the optimal solution of the maximum evolution iteration.

\section{Simulation Experiments}

In planar space manipulator movement, for example, the carrier and the mechanical arm to complete the set time $\mathrm{T}=55$. The genetic algorithm control parameters were set as: $\mathrm{M}=48$ group size, the length of the chromosome N. 2:10, crossover probability $p_{c}=0.8$, the mutation probability $p_{m}=0.06$, the evolution algebra $\mathrm{R}=200$. Carrier set $\pi / 4$ of initial and terminal $\pi / 2$ configuration remains the same, before the mechanical arm lever shifted from initial configuration $\pi / 6$ to end configuration $\pi / 3$, initial configuration to the terminal at the end of the rod by configuration. The initial $x_{0}=[0, \pi / 4, \pi / 6]^{T}$ and terminal configuration $x_{1}=[0, \pi / 2, \pi / 3]^{T}$ set system respectively, and two aspects of the selection and Angle of minimax to measure, the results as shown in Figure 1 and Figure 2.

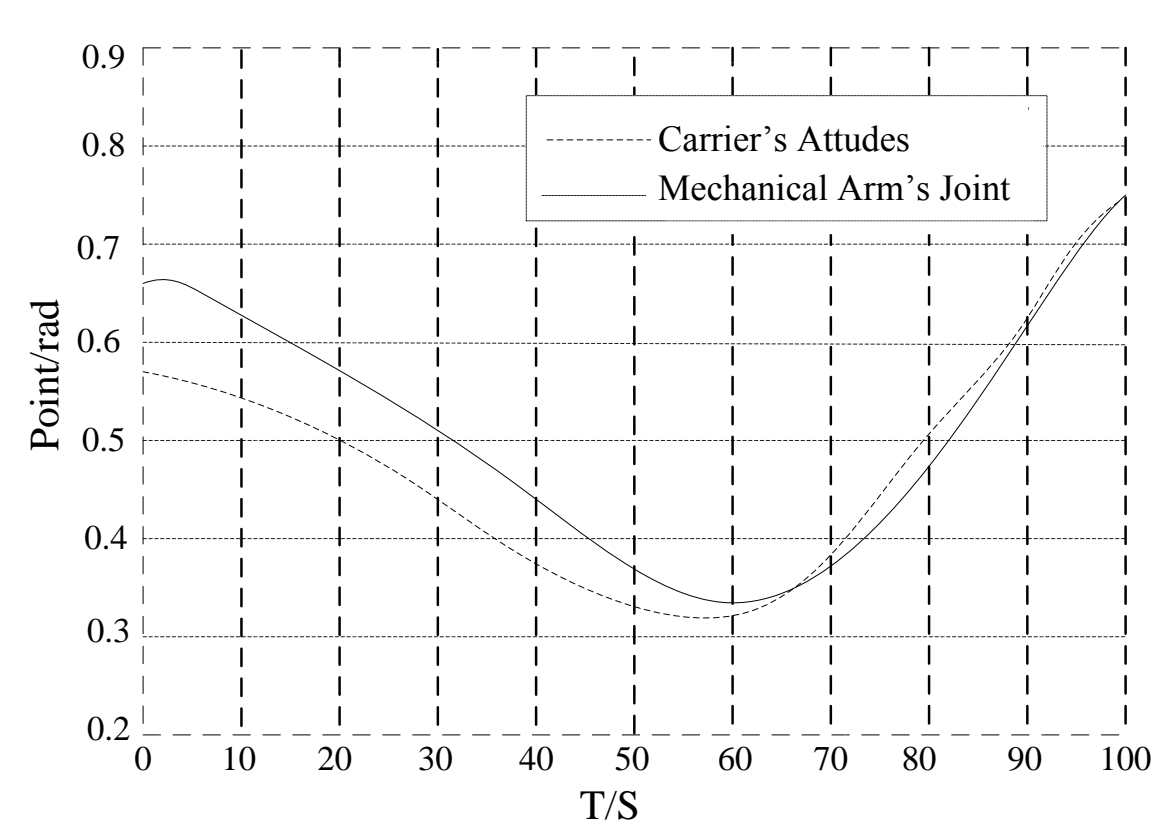

Figure 1. Changes with the Angle Ranging $[0.2,0.9]$ 


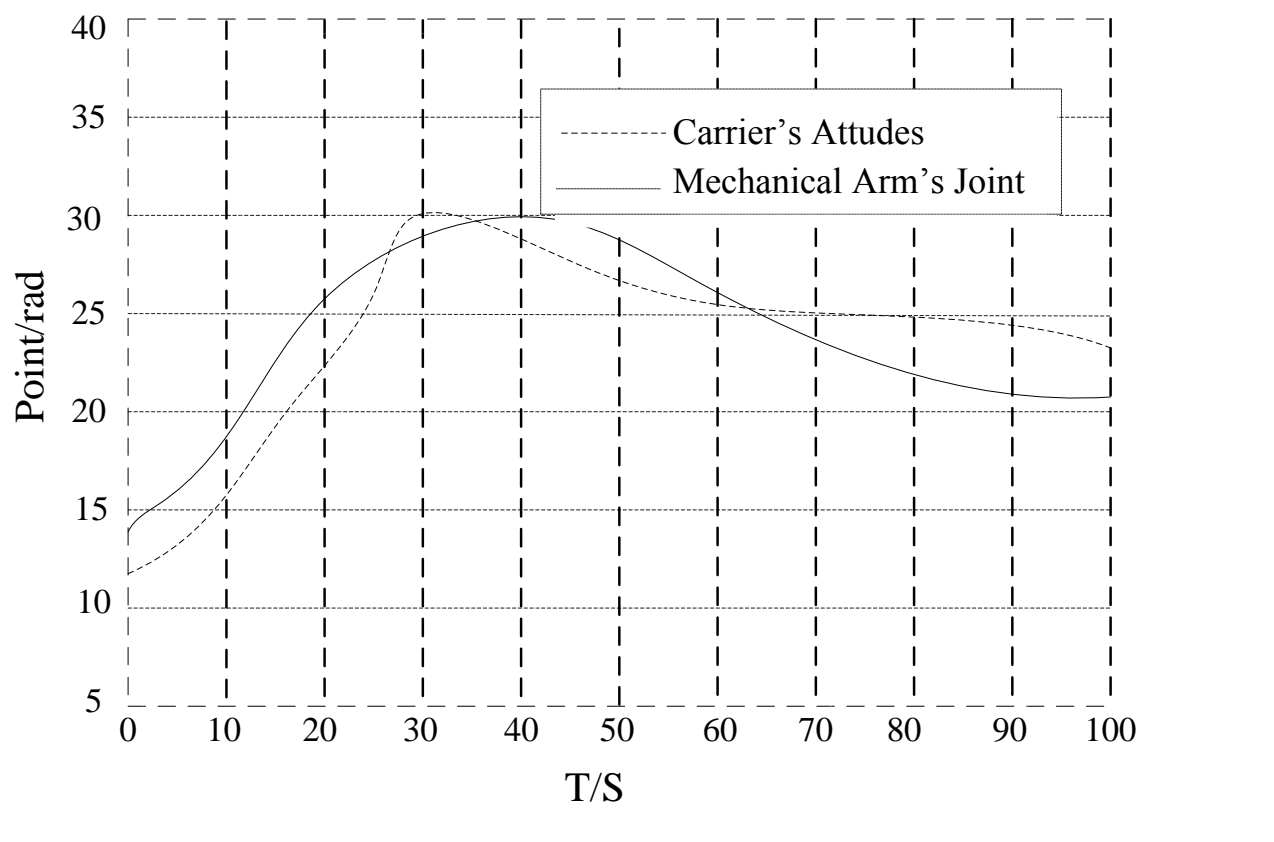

Figure 2. Changes with the Angle Ranging [5-40]

\section{Conclusion}

This paper first analyzes motions of mechanícal arms, and then applied neutral network in the analysis of mechanical arm's motions Finally, it analyzes the motion of the space manipulator, and then uses the neural network to analyze the manipulator motion. The simulation experiment shows that the method is effective and can improve the movement effect of the robot âtm.

\section{References}

[1] X.-P. Shi and S.-R. Liu "A Sulrvey of Trajectory Tracking Control for RobotM an inpulators", Control Engineering of China vol. 18, no. 1, (2011), pp. 116-121.

[2] C.-H. Qian, S. Zhen and B. Yang, "Design and research for comfort control algorithm based on somatosensory robotic arm", Application of Electronic Technique, vol. 41, no. 9, (2015), pp. 67-69.

[3] K.-Z. Lv, S.-X. Chen and Q.-Z. Huang, "Flexible manipulator trajectory tracking and vibration fuzzy control', Chinese Journal of Engineering Design, vol. 22, no. 1, (2015), pp. 78-82.

[4] C. Jie and M, Wei, "Adaptive fuzzy sliding mode control for crawler \} ype mobile manipulators", Journal of Jilin University(Engineering and Technology Edition), vol. 45, no.3, (2015), pp. 892-898.

[5] Q. H. Dong and C. Li, "Neural network stabilization control of space manipulator capturing operation", Journal of Huazhong University of Science and Technology(Natural Science Edition), vol. 43, no. 3, (2015), pp. 22-26.

[6] Q.-H. Dong and C. Li, “Adaptive Control Simulation of Space Manipulator Capturing Target”, Journal of System Simulation, vol. 26, no. 12, (2014), pp. 2969-2973.

[7] C. Wang, Z.-H. Dong and Y.-M. Gao, "Control Strategy for Space Manipulator Based on Genetic Algorithm Self-Tuning”, Fire Control \& Command Control, (2014), vol. 39, o. 2, pp. 61-63.

\section{Fund Project}

Quzhou City guidance of science and technology plan projects (2015014) 


\section{Authors}

Wenjun Xu(1981.09-), he is a lecturer, master, research direction: Material Formation and Control Engineering

Lan Chen(1983.08-), he is an Engineer, master, research direction: machine design

Yuzhen Lin(1984.10-), he is a lecturer, master, research direction: machine design

Wenjun Liu(1972.07-), he is an associate professor, bachelor, research direction: Digit Control Technique

Fan Yang(1976.02-), he is an associate professor, master, research directiop: machine design

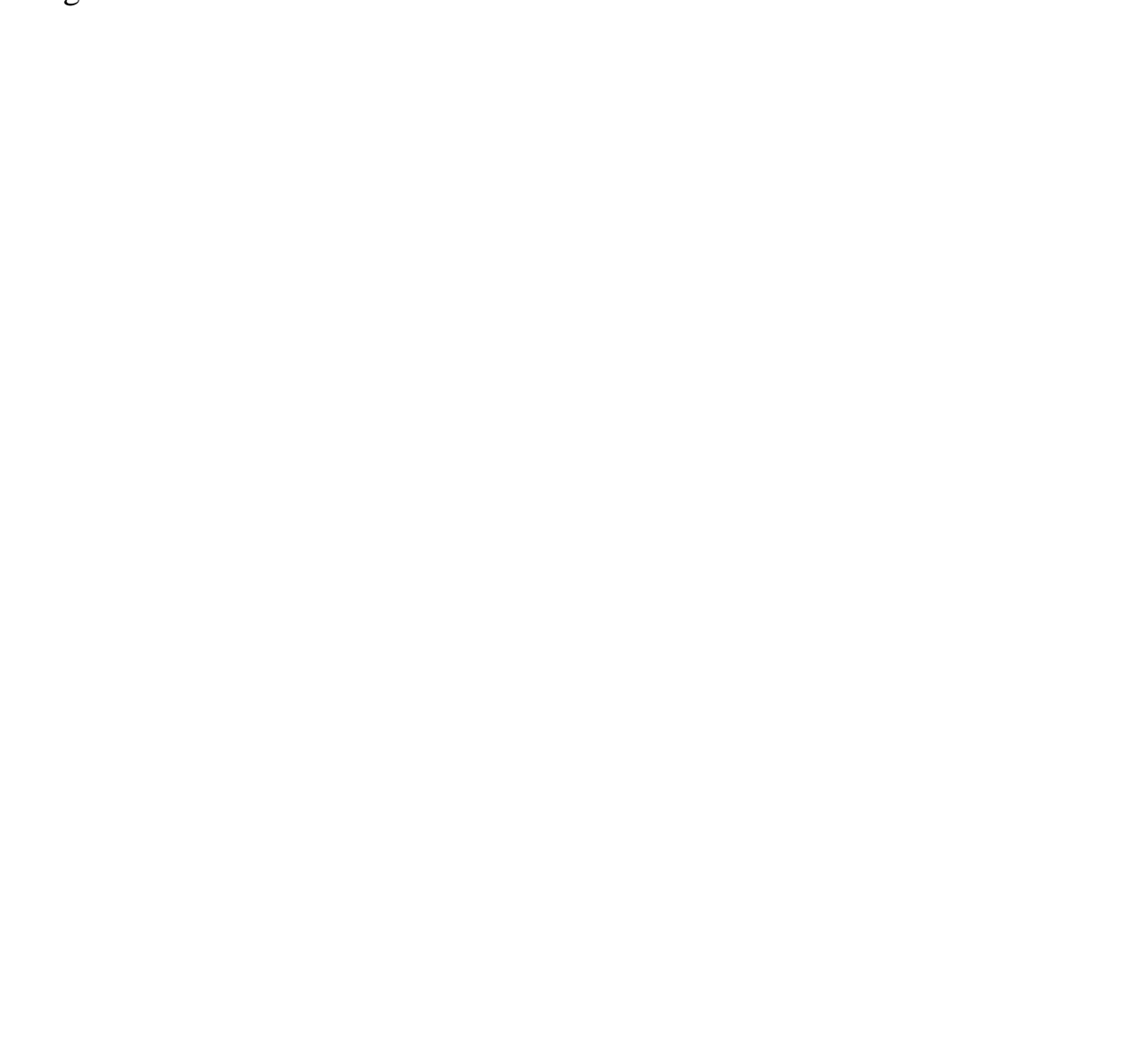


International Journal of Control and Automation

Vol. 9, No. 8 (2016)

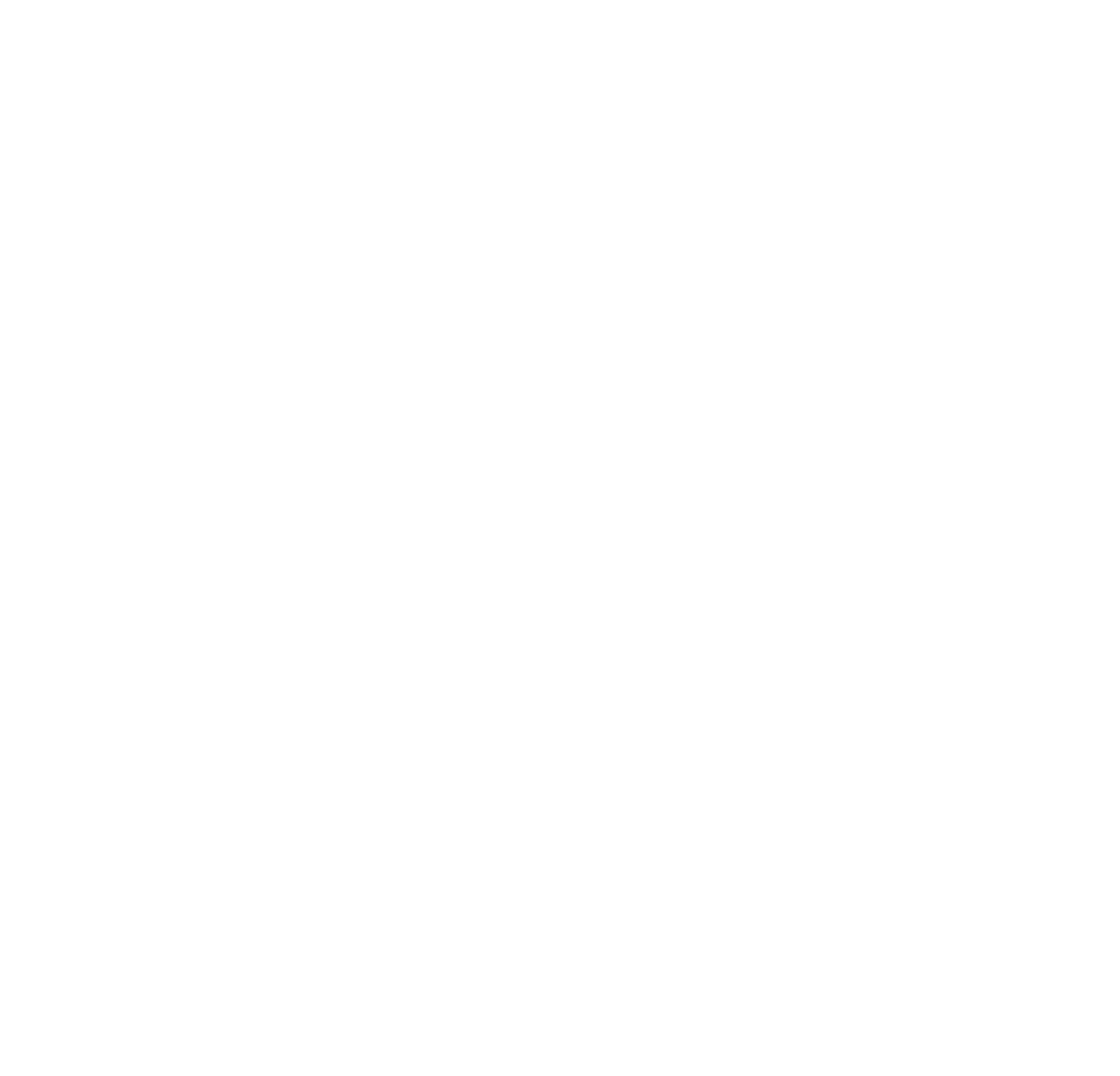

